Новрузбеков М.С. *, Олисов О.Д., Гуляев В.А., Луцык К.Н., Магомедов К.М.

ГБУЗ “Научно-исследовательский институт скорой помощи им. Н.В. Склифосовского Департамента здравоохранения г. Москвы”; 129090, Москва, Большая Сухаревская пл., д. 3, Российская Федерация

Цель. Оценка результатов лечения пациентов, перенесших ортотопическую трансплантацию и экстракорпоральную резекцию печени ex situ, ex vivo по поводу ее нерезектабельного поражения.

Материал и методы. Выполнено 22 ортотопические трансплантации печени, 4 экстракорпоральные резекции и аутотрансплантации органа ex situ. Трансплантация выполнена 10 пациентам с нерезектабельной гепатоцеллюлярной карциномой, 8 - с нерезектабельным альвеококкозом, 4 - с эпителиоидной гемангиоэндотелиомой. Экстракорпоральная резекция выполнена 3 пациентам с альвеококкозом, 1 - с холангиоцеллюлярным раком.

Результаты. Послеоперационные осложнения развились у 38,4\% больных, летальность составила $19,2 \%$. Наибольшее число летальных исходов отмечено среди пациентов с альвеококкозом - 36,3\%. В группе пациентов с гепатоцеллюлярной карциномой летальность составила $10 \%$, среди пациентов с гемангиоэндотелиомой и холангиоцеллюлярным раком летальных исходов не было. Медиана общей выживаемости для пациентов с гепатоцеллюлярным раком, альвеококкозом и гемангиоэндотелиомой составила 48, 36 и 20 мес соответственно. Пациент, перенесший экстракорпоральную резекцию в связи с холангиоцеллюлярным раком, жив в течение 24 мес.

Заключение. Трансплантация и аутотрансплантация печени - единственный вариант радикального лечения при нерезектабельном альвеококкозе и некоторых онкологических заболеваниях органа. Пациенты с нерезектабельным альвеококкозом печени представляют группу повышенного риска послеоперационных осложнений и летальности в связи с продолжительностью заболевания, плохим физическим статусом, хронической инфекцией и паразитарной инвазией.

Ключевые слова: печень, трансплантация, альвеококкоз, гепатоцеллюлярная карцинома, аутотрансплантация печени, гемангиоэндотелиома, отдаленные результаты.

Ссылка для цитирования: Новрузбеков М.С., Олисов О.Д., Гуляев В.А., Луцык К.Н., Магомедов К.М. Трансплантация и аутотрансплантация как радикальный метод лечения при нерезектабельных опухолевых и паразитарных заболеваниях печени. Анналы хирургической гепатологии. 2020; 25 (4): 49-59. https://doi.org/10.16931/1995-5464.2020449-59.

Авторы подтверждают отсутствие конфликтов интересов.

\title{
Transplantation and autotransplantation of the liver in radical treatment of unresectable liver tumors and parasitic diseases
}

\author{
Novruzbekov M.S. *, Olisov O.D., Guliaev V.A., Lutsyk K.N., Magomedov K.M. \\ Sklifosovsky Research Institute for Emergency Medicine; 3/5, Bolshaya Suharevskaya sq., Moscow, 129090, \\ Russian Federation
}

\footnotetext{
Aim. To evaluate results of liver transplantation and ex situ liver resection and autotransplantation in patients with unresectable parasitic and tumor liver lesions.

Matherial and methods. A total of 22 orthotopic liver transplants and 4 ex situ liver resection and autotransplantations were performed. Liver transplants performed in 10 cases of unresectable hepatocellular carcinoma, 8 cases of alveolar echinococcosis, 4 cases of hepatic epithelioid haemangioendothelioma. Ex situ liver resection and autotransplantation were performed in 3 cases of alveolar echinococcosis and in 1 case of cholangiocarcinoma.

Results. Postoperative complication developed in 38,4\% patients. Mortality rate was $19.2 \%$. Patients with alveolar echinococcosis were most complicated group with $36,3 \%$ early mortality rate. Mortality in hepatocellular carcinoma group was $10 \%$. There were no early mortality in haemangioendothelioma and cholangiocarcinoma patients. Median
} 
overall survival for hepatocelluler carcinoma, alveolar echinococcosis and hepatic epithelioid haemangioendothelioma groups was 48, 36, 20 months respectively. Patient after ex situ liver resection for cholangiocarcinoma alive for 24 months and still disease free.

Conclusion. Liver transplantation and ex situ liver resection and autotransplantation is the only opportunity for radical treatment for unresectable alveolar echinococcosis and some oncological diseases of the organ. Patients with unresectable alveolar echinococcosis is a high risk group of postoperative complications due to initial poor physical status, long-time disease, chronically infections and parasitic invasion.

Keywords: liver, transplantation, alveolar echinococcosis, hepatocellular carcinoma, liver autotransplantation, hepatic epithelioid haemangioendothelioma, long-term results.

For citation: Novruzbekov M.S., Olisov O.D., Guliaev V.A., Lutsyk K.N., Magomedov K.M. Transplantation and autotransplantation of the liver in radical treatment of unresectable liver tumors and parasitic diseases. Annaly khirurgicheskoy gepatologii $=$ Annals of HPB surgery. 2020; 25 (4): 49-59. (In Russian). https://doi.org/10.16931/1995-5464.2020449-59.

There is no conflict of interests.

\section{Введение}

Резекция печени является (РП) основным методом лечения при ее очаговых поражениях. Первыми из важнейших требований, которым должна отвечать РП, - переносимость и безопасность для пациента. Следующим по важности требованием является эффективность РП, а именно ее радикальность. В силу объективных обстоятельств эти требования не всегда могут быть соблюдены. Нерезектабельность очагового поражения печени, как правило, обусловлена ее недостаточными функциональными резервами, малым объемом интактной паренхимы или местным распространением патологического процесса с вовлечением магистральных сосудов печени [1]. Очевидно, что РП в таких условиях или невыполнима, или заведомо обречена на неудачу. Ортотопическая трансплантация печени (ОТП) является именно тем вариантом лечения, который характеризуется максимальной радикальностью и востребован при некоторых нерезектабельных поражениях печени, а также способен предоставить гарантии длительной выживаемости и нормального качества жизни. В ряде наблюдений при ограниченном поражении паренхимы печени с вовлечением магистральных печеночных вен экстракорпоральная резекция и аутотрансплантация печени (ЭРиА) может конкурировать с ОТП по степени радикальности и качеству отдаленных результатов.

\section{Материал и методы}

С 2007 по 2019 г. в Московском городском центре трансплантации печени оперированы 26 пациентов с первично нерезектабельной формой очагового поражения печени. Гепатоцеллюлярная карцинома (ГЦК) выявлена у $10(38,4 \%)$ больных, альвеококкоз печени (АП) -у 11 (42,3\%), эпителиоидная гемангиоэндотелиома (ЭГ) у $4(15,4 \%)$, холангиоцеллюлярный рак (ХЦР) у $1(3,9 \%)$. Медиана возраста пациентов составила 36,5 лет (21-60 лет; ДИ (25-75\%) 21-62 года);
13 (50\%) больных были младше 35 лет. Обследование пациентов поводили по программе ТП, включавшей стандартный набор исследований (лабораторные методы, эндоскопическое исследование желудочно-кишечного тракта, исследование функции сердечно-сосудистой и дыхательной систем), рутинные радиологические исследования (УЗИ, МРТ, КТ грудной клетки и брюшной полости, сцинтиграфия скелета). Для исключения внепеченочного распространения дополнительно выполняли диагностическую видеолапароскопию $(n=4)$ и видеоторакоскопию $(n=2)$.

Оперативное лечение было представлено двумя видами хирургических вмешательств: ОТП выполнена в 22 наблюдениях, ЭРиА - в 4. Выбор хирургической тактики осуществляли по объему поражения печени, нозологической форме заболевания, наличию отдаленных метастазов, а также специфике региональной политики органного донорства, регламентируемой законодательством РФ. При наличии достаточного объема анатомически и функционально сохранной паренхимы печени $(\geqslant 30 \%)$, четкой уверенности в технической возможности восстановления афферентного и эфферентного кровотока, а также ввиду отсутствия юридической возможности включить пациента в лист ожидания трупной ТП, выполняли ЭРиА ex situ, ex vivo (рис. 1). В остальных наблюдениях ОТП рассматривали как операцию выбора.

ЭРиА включала гепатэктомию с резекцией нижней полой вены (НПВ), восстановление оттока по НПВ (протезирование гофрированным сосудистым протезом или трупным венозным аллотрансплантатом), формирование временного портокавального шунта, собственно экстракорпоральную РП с протезированием магистральных печеночных сосудов в условиях холодовой перфузии (такназываемыйэтап “back-table” или “on bench") и последующую имплантацию органа (рис. 2-6). 


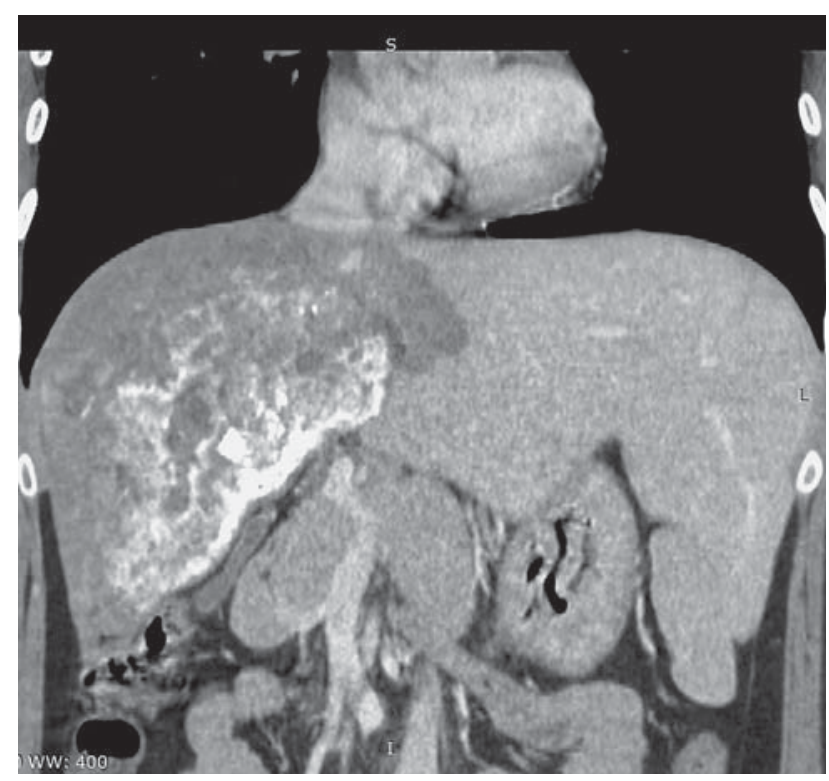

Рис. 1. Компьютерная томограмма. Альвеококкоз печени с поражением правой доли, IVa сегмента и устья печеночных вен.

Fig. 1. Computed tomography. Liver alveolar echinococcosis with involving right lobe, SIV-a and hepatic veins.

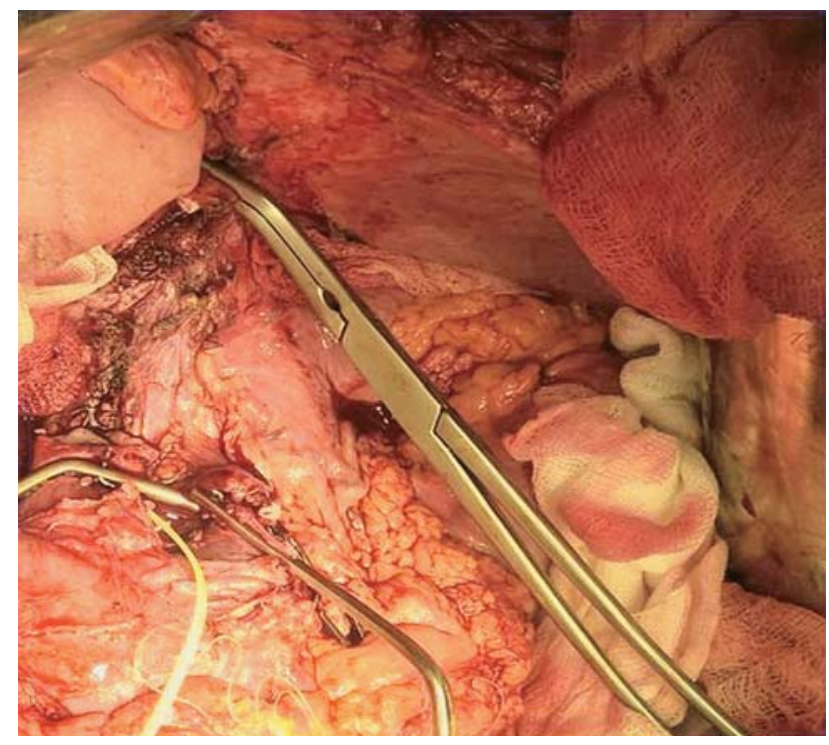

Рис. 3. Интраоперационное фото. Гепатэктомия с резекцией НПВ и правого купола диафрагмы.

Fig. 3. Intraoperative photo. Total hepatectomy with IVC and right hemidiaphragm resection.

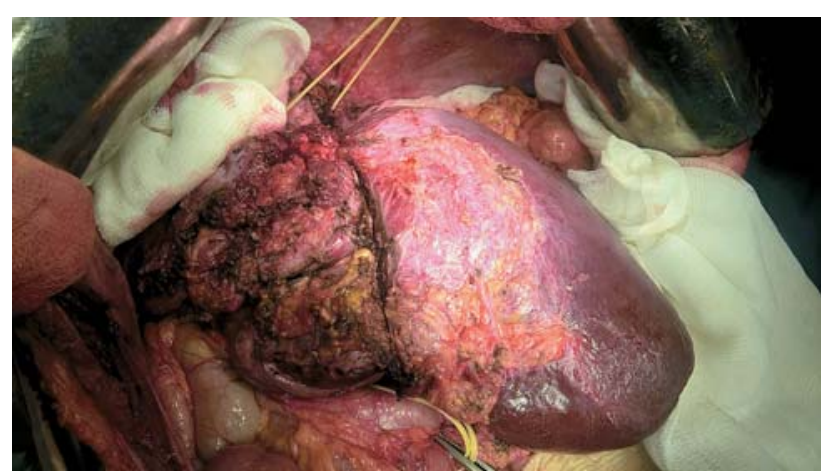

Рис. 2. Интраоперационное фото. Альвеококкоз IV, V, VIII сегментов печени с поражением правой и общего ствола средней и левой печеночных вен и прорастанием правого купола диафрагмы; 12 лет после паллиативной резекции правой доли.

Fig. 2. Intraoperative photo. Alveolar echinococcosis of the IV, V, VIII liver segments involving right diaphragm and all hepatic veins. 12 years after right lobe palliative resection.

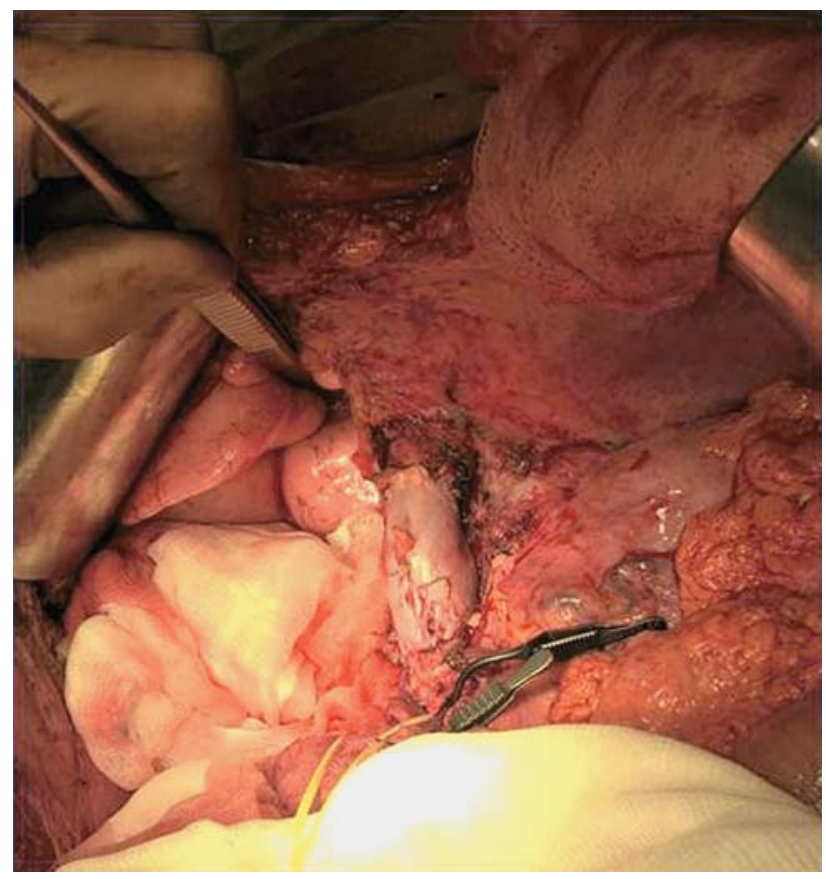

Рис. 4. Интраоперационное фото. Этап протезирования НПВ венозным аллотрансплантатом.

Fig. 4. Intraoperative photo. IVC reconstructed stage with a venous allograft. 


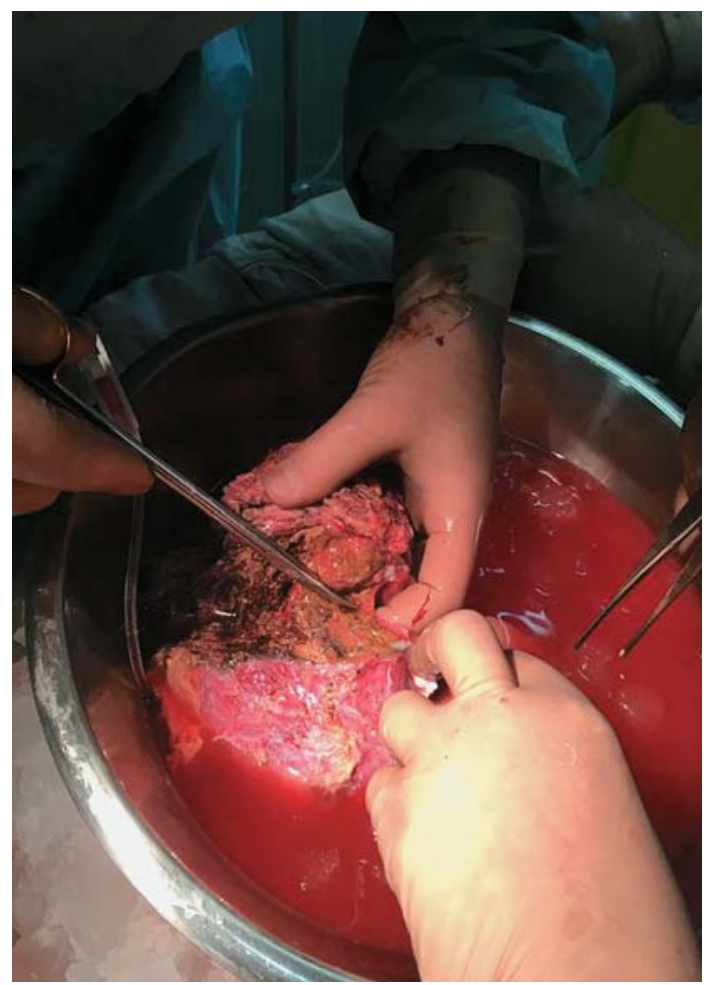

Рис. 5. Интраоперационное фото. Экстракорпоральная резекция в условиях холодовой перфузии, этап "backtable".

Fig. 5. Intraoperative photo. Hypothermic extracorporal liver resection, "black-table" stage.

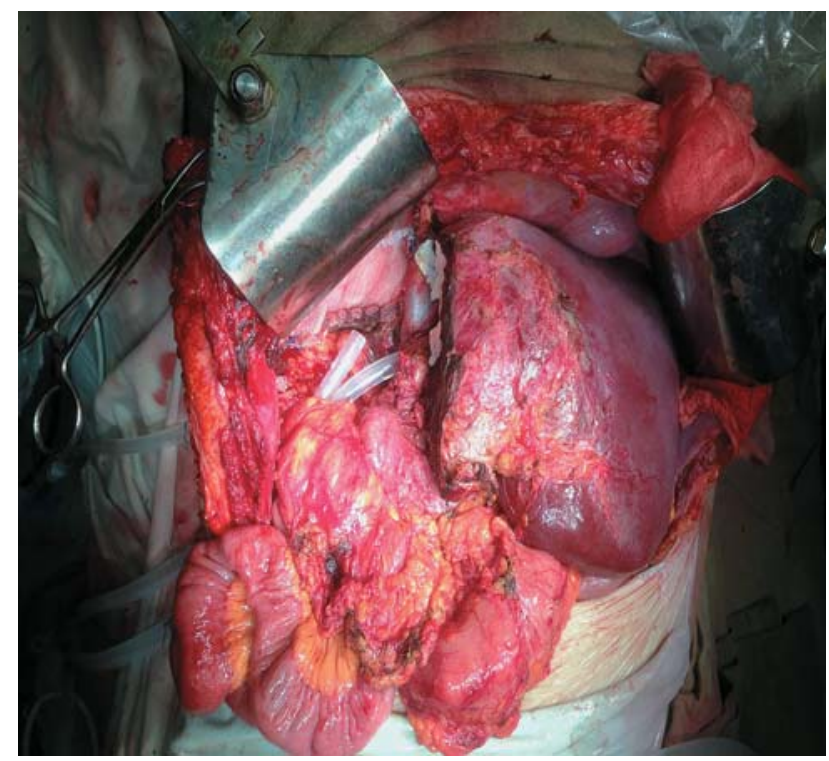

Рис. 6. Интраоперационное фото. Этап имплантации левого латерального сектора печени, дефект диафрагмы замещен сетчатым эндопротезом.

Fig. 6. Intraoperative photo. Left lateral sector implantation stage. Diaphragm reconstructed with endoprothesis.

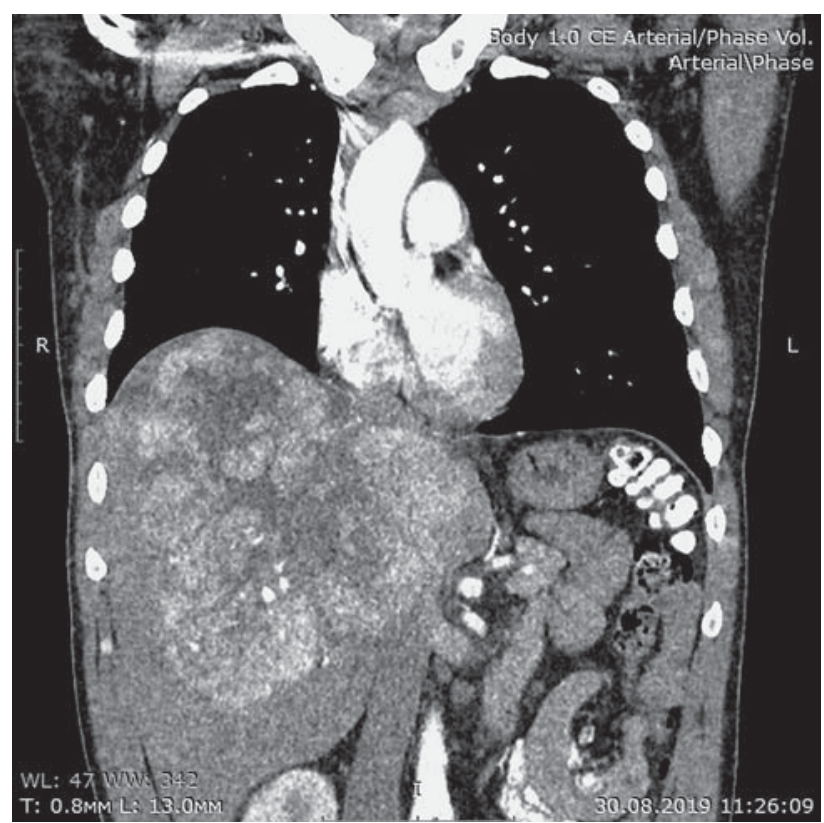

Рис. 7. Компьютерная томограмма. Печеночноклеточный рак, тотальное поражение органа.

Fig. 7. Computed tomography. Hepatocellular carcinoma, total lesion.

У всех пациентов с ГЦК опухоль развилась вне фонового диффузного заболевания печени. Семь (70\%) пациентов были мужского пола. В 6 (60\%) наблюдениях наиболее вероятной причиной развития рака послужило продолжительное употребление половых гормонов. В 4 наблюдениях установить причину развития ГЦК не удалось. У 9 (90\%) больных внепеченочное распространение исключено по результатам предоперационного обследования. У одной пациентки интраоперационно выявлен солитарный имплантационный метастаз в переднюю брюшную стенку. Всем пациентам из группы ГЦК выполнена ОТП, поскольку объем поражения печени не предполагал иного радикального лечения (рис. 7). В 9 (90\%) наблюдениях гепатэктомия выполнена с сохранением НПВ, в 1 наблюдении - с ретропеченочным отделом НПВ.

Четверо $(36,4 \%)$ из 11 пациентов с АП ранее перенесли паллиативные вмешательства. Медиана продолжительности заболевания составила 4 года (от 1 до 17 лет, в 3 наблюдениях >10 лет). В $9(81,8 \%)$ из 11 наблюдений альвеококкоза выявлена паразитарная инвазия в магистральные сосуды и окружающие ткани (табл. 1, рис. 8). В 1 наблюдении отмечено билобарное метастатическое поражение легких.

ОТП выполнена в 8 (72,7\%) из 11 наблюдений, при этом гепатэктомия в “классическом" варианте потребовалась 5 (62,5\%) больным. ЭРиА 
Таблица 1. Формы паразитарной инвазии при нерезектабельном АП

Table 1. Types of parasitic invasion in unresectable liver alveolar echinococcosis

\begin{tabular}{l|c}
\hline \multicolumn{1}{c|}{ Характер инвазии } & Число наблюдений, абс. (\%) \\
\hline НПВ & $3(33,3)$ \\
НПВ и диафрагма & $1(11,1)$ \\
НПВ, воротная вена и печеночная артерия & $2(22,2)$ \\
НПВ, воротная вена и печеночная артерия, ЖКТ & $2(22,2)$ \\
НПВ (тромбоз до уровня подвздшных вен), воротная вена, & $1(11,1)$ \\
диафрагма, перикард & \\
\hline
\end{tabular}

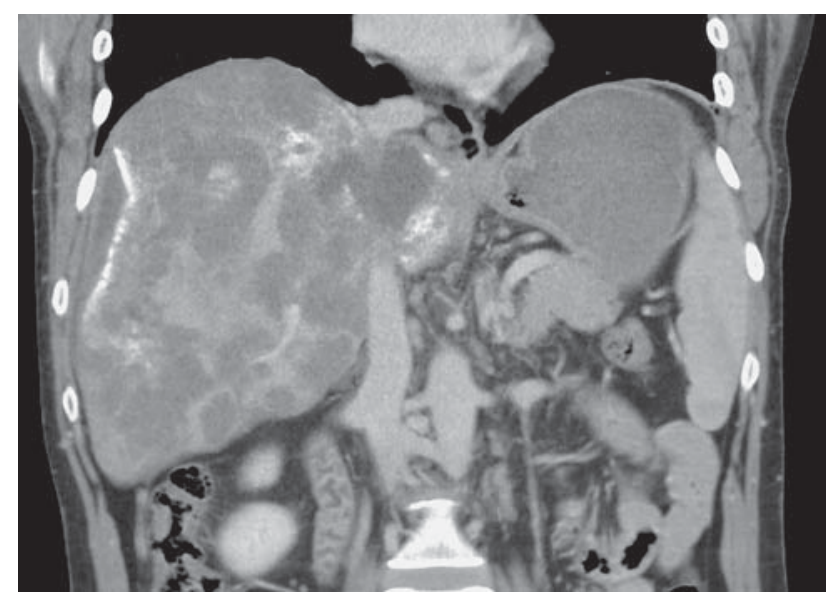

Рис. 8. Компьютерная томограмма. Альвеококкоз печени, тотальное поражение с прорастанием правого купола диафрагмы.

Fig. 8. Computed tomography. Total liver and right diaphragm alveolar echinococcosis.

выполнена в $3(27,3 \%)$ наблюдениях, во всех наблюдениях потребовалась резекция ретропеченочного отдела НПВ.

ЭГ диагностирована у 4 пациентов, 3 (75\%) из них были младше 30 лет (23-28 лет). Во всех наблюдениях отмечено тотальное поражение пече-

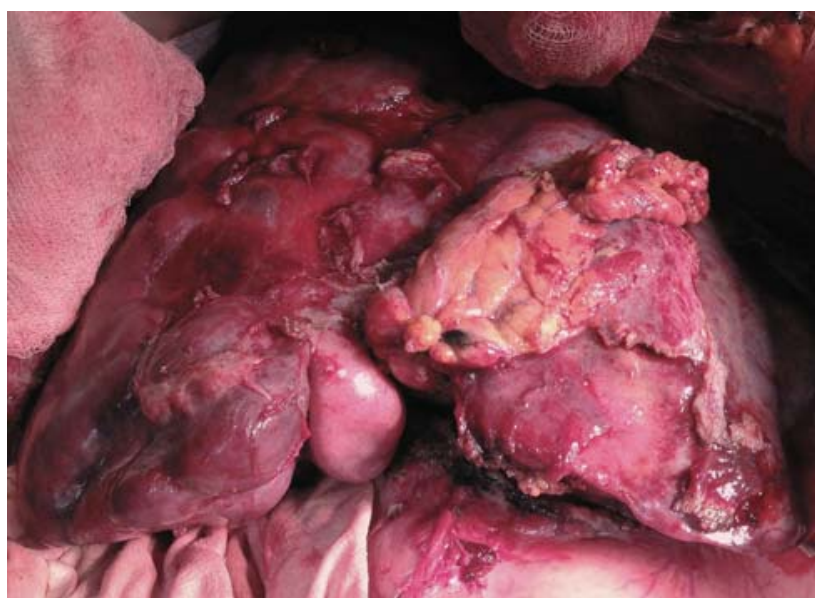

Рис. 9. Интраоперационное фото. Эпителиоидная гемангиоэндотелиома, тотальное поражение печени.

Fig. 9. Intraoperative photo. Hepatic epithelioid haemangioendothelioma, total lesion of the liver. ни, исключавшее какой-либо вариант ее резекции (рис. 9). Гистологическая верификация диагноза до операции была у 3 пациентов. Данные предоперационного гистологического исследования не противоречили включению пациентов в лист ожидания ТП, поскольку клеточная атипия и митотическая активность опухоли были низкими. Всем пациентам группы выполнена ОТП с сохранением ретропеченочного отдела НПВ.

У пациентки с ХЦР опухоль локализовалась в зоне конфлюенса печеночных вен с инвазией в НПВ (рис. 10). До операции больная перенесла 5 курсов полихимиотерапии и курс стереотаксической терапии. Достаточный объем интактной и функционально состоятельной паренхимы, благоприятные технические условия для восстановления кровоснабжения печени, а также природа основного заболевания, характеризующаяся исключительной агрессивностью, послужили причиной выбора ЭРиА как метода хирургического лечения.

\section{Результаты}

У 16 (72,7\%) больных, перенесших ОТП, осложнений не отмечено. Послеоперационные осложнения отмечены у $10(38,4 \%)$ из 26 пациентов - у $6(27,3 \%)$ после ОТП и у $4(100 \%)$ после

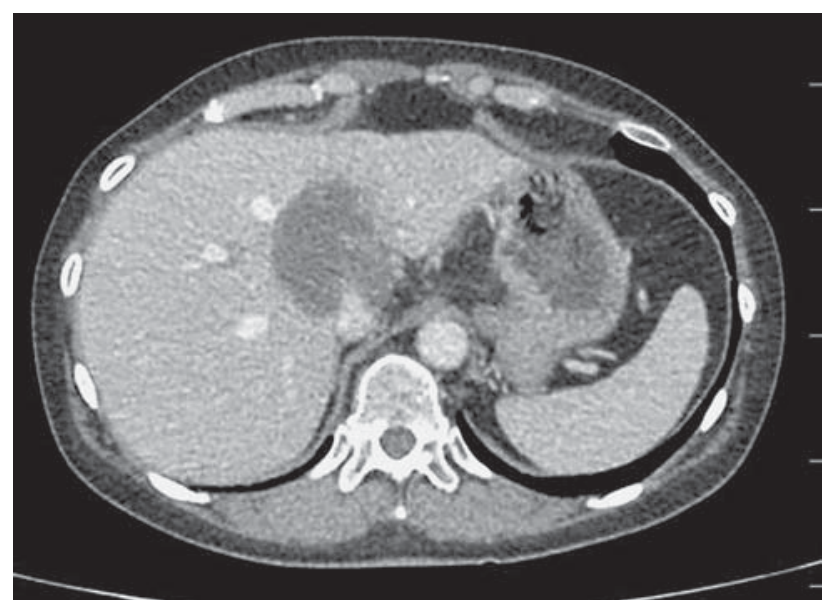

Рис. 10. Компьютерная томограмма. Холангиоцеллюлярный рак, поражение кавальных ворот печени.

Fig. 10. Computed tomography. Cholangiocarcinoma of the liver involving all hepatic veins. 
Таблица 2. Характер осложнений после хирургического лечения

Table 2. Postoperative complications after surgical treatment

\begin{tabular}{|c|c|c|c|c|}
\hline \multirow[t]{2}{*}{ Осложнение } & \multicolumn{2}{|c|}{ Число наблюдений, абс. } & \multicolumn{2}{|c|}{$\begin{array}{c}\text { Число летальных } \\
\text { исходов, абс. (\%) }\end{array}$} \\
\hline & ОТП & ЭРиА & ОТП & ЭРиА \\
\hline Острое клеточное отторжение трансплантата & 1 & & - & - \\
\hline Тромбоз левой печеночной артерии и вены & 1 & & - & - \\
\hline Тромбоз печеночной артерии & 1 & & $1(100)$ & - \\
\hline Сепсис & 3 & & $3(100)$ & - \\
\hline Энцефалопатия & & 1 & - & - \\
\hline Послеоперационная лимфорея & & 1 & - & - \\
\hline Внутрибрюшное кровотечение & & 1 & - & - \\
\hline Печеночная недостаточность & & 1 & - & $1(100)$ \\
\hline
\end{tabular}

ЭРиА. Ранняя послеоперационная летальность составила 19,2\% (табл. 2). Ближайшие и отдаленные результаты существенно отличались в нозологических группах, поэтому результаты будут рассмотрены отдельно для каждой группы.

Гепатоцеллюлярная карцинома. Послеоперационные осложнения наблюдались у 2 пациентов с ГЦК и были связаны с нарушением кровообращения в трансплантате. В одном наблюдении тромбоз в бассейне левой печеночной артерии и вены, развившийся на 4-е сутки, привел к тяжелой печеночно-почечной недостаточности, потребовавшей включения пациента в экстренный лист ожидания ретрансплантации печени. В период ожидания нового донорского органа проводили комплексную интенсивную терапию, и к исходу 10 дней лечения отмечено восстановление кровотока в пораженной доле печени. В другом наблюдении тромбоз общей печеночной артерии инициировал каскад необратимых патологических процессов (некроз желчных протоков, печеночная недостаточность), повлекших утрату трансплантата и смерть пациента. В сложившейся ситуации единственным вариантом лечения была экстренная и своевременная ретрансплантация, однако ни в первом, ни во втором наблюдении донорского органа в необходимые сроки не было. Таким образом, показатель ранней послеоперационной летальности в группе пациентов с ГЦК составил 10\% (1 из 10 пациентов).

Альвеококкоз печени. Ранние послеоперационные осложнения диагностированы у 5 (45\%) пациентов с АП, умерли 4 (36,3\%) пациента. В 3 наблюдениях у перенесших ОТП развился сепсис, которому способствовали хроническая билиарная инфекция, инфицированные паразитарные кисты, алиментарная недостаточность. Травматичность оперативных вмешательств у пациентов с АП была существенно больше по сравнению со стандартной процедурой ОТП, усугублялась паразитарной инвазией и тяжелым спаечным процессом у ранее (порой неоднократ- но) оперированных пациентов. Четырем (50\%) пациентам, перенесшим ТП, потребовалась резекция смежных органов: правого купола диафрагмы, желудка, ободочной кишки, перикарда. Летальность в группе перенесших ТП по поводу альвеококкоза составила $37,5 \%$.

В группе ЭРиА осложнения отмечены у всех пациентов $(n=3)$. Гипокоагуляция и обширная раневая поверхность резецированной печени послужили причиной внутрибрюшного кровотечения и длительной лимфореи у 2 больных. Послеоперационная печеночная недостаточность развилась в 1 наблюдении и явилась результатом своевременно не диагностированного фиброза, развившегося на фоне длительной кавальной обструкции. Летальность в группе составила $33,3 \%$.

У 4 пациентов, перенесших ТП по поводу ЭГ, осложнений в раннем послеоперационном периоде не отмечено. И, наконец, еще в 1 наблюдении ЭРиА у пациентки с ХЦР отмечена непродолжительная послеоперационная энцефалопатия, обусловленная, по-видимому, совокупностью таких факторов, как обширная резекция, холодовая ишемия и полихимиотерапия, предшествовавшая оперативному вмешательству. Таким образом, в двух последних группах ранней послеоперационной летальности не было.

Отдаленные результаты. Медиана общей выживаемости (OB) в совокупной популяции оперированных пациентов составила 39 мес (рис. 11). Сроки наблюдения за пациентами из группы ГЦК составили 12-152 мес. Прогрессирование ГЦК диагностировано у 3 (33\%) из 9 пациентов. Медиана безрецидивной выживаемости (БРВ) составила 40 мес (4-108 мес), медиана ОВ - 48 мес (12-152 мес). У 44,4\% пациентов срок наблюдения превышает 5 лет. Все 9 пациентов к настоящему времени живы, социально и физически адаптированы, пациенты с прогрессированием ГЦК получают специализированное онкологическое лечение и продолжают работать по своей основной специальности. 
Рис. 11. Диаграмма. Общая выживаемость после хирургического лечения.

Fig. 11. Diagram. Overall survival after surgery.

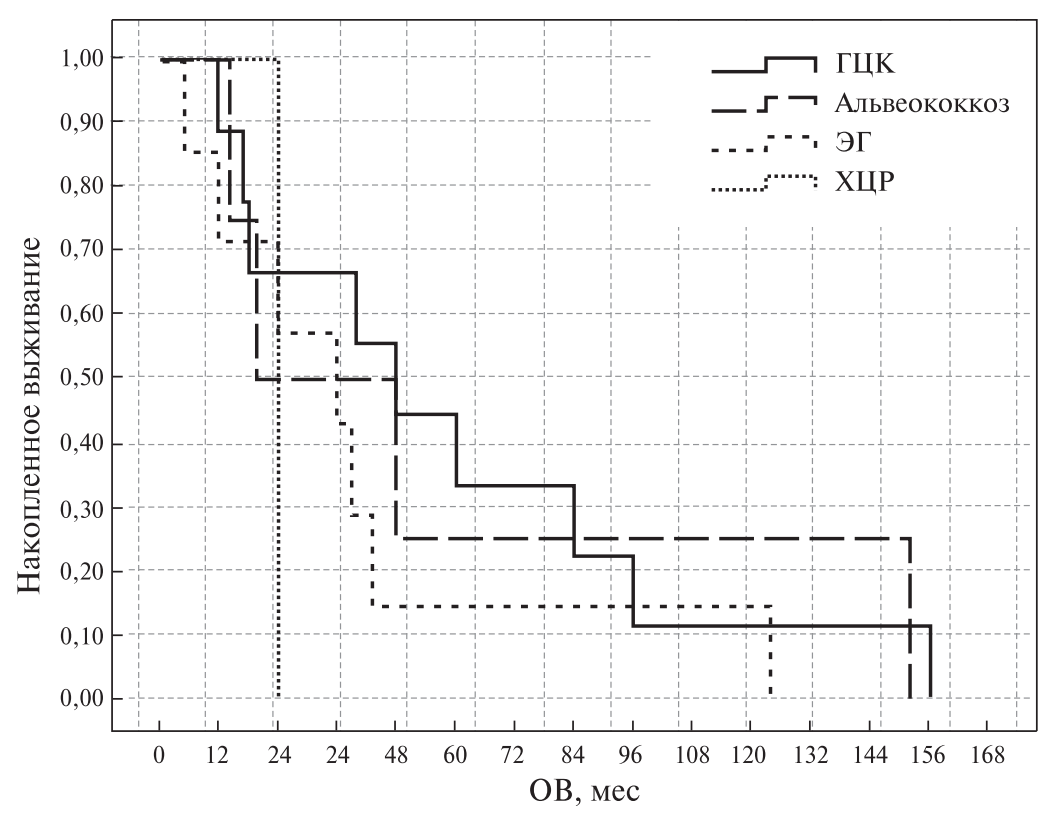

В группе пациентов, оперированных по поводу АП, сроки наблюдения составляют 5-124 мес, медиана ОВ - 36 мес. Рецидив отмечен в 1 наблюдении и потребовал повторного оперативного вмешательства (правосторонняя адреналэктомия) спустя 2 года после ОТП.

Из 4 пациентов, трансплантированных по поводу ЭГ, рецидив заболевания отмечен у половины, сроки наступления рецидива в обоих наблюдениях составили 4 мес. Оба пациента умерли от прогрессирования ЭГ через 14 и 20 мес после операции. Два пациента живы, признаков рецидива нет, сроки наблюдения составляют 48 и 152 мес. Медиана ОВ пациентов, перенесших ТП по поводу ЭГ, составила 20 мес.

Пациентку, перенесшую ЭРиА по поводу ХЦР, наблюдаем 24 мес, данных о прогрессировании заболевания нет.

\section{Обсуждение}

Нерезектабельные очаговые поражения печени занимают весьма скромное место в структуре показаний к ОТП, что можно объяснить комплексом причин. Подобные заболевания не имеют большого распространения, поэтому их доля в общей популяции перенесших ТП невелика. ГЦК, развившаяся в отсутствие фоновых заболеваний печени, таких как хронический гепатит и цирроз, составляет не более $10 \%$ от всех наблюдений печеночноклеточного рака, ЭГ - не более $1 \%$ от всех злокачественных поражений печени, а альвеококкоз характеризуется эндемичностью - чаще всего его выявляют в Сибири, на Дальнем Востоке, в Китае, Средней Азии [2-6]. Другой причиной является ограниченная доступность ТП большинству таких пациентов в силу сохраняющегося дефицита донорских органов, обоснованных опасений малой БРВ и ОВ, а также в связи с пока еще недостаточным объемом трансплантационной помощи в регионах. Вместе с тем существующие рекомендации достаточно четко определяют целесообразность ОТП пациентам с нерезектабельными очаговыми поражениями печени. При местнораспространенном АП, ЭГ и ГЦК, развившейся вне фонового заболевания, показанием к ОТП будет собственно нерезектабельность опухоли и отсутствие внепеченочного распространения [2-6].

При тщательном отборе пациентов пятилетняя ОВ после ТП при нерезектабельной ГЦК составляет не менее 50\% [5]. Наши результаты вполне сопоставимы с этими данными: однолетняя, трех- и пятилетняя ОВ составляют 90, 60 и 45\%. Рассчитываем на улучшение этих показателей, поскольку сроки наблюдения за некоторыми пациентами приближаются к трем и пяти годам. С учетом того что эти пациенты были признаны бесперспективными для хирургического лечения, а эффективность системной терапии при таком объеме поражения сомнительна, полученными результатами вполне удовлетворены.

Не являясь онкологическим заболеванием по своей природе, АП характеризуется бессимптомным течением на ранних стадиях, медленным, но инфильтративным ростом и тенденцией к метастазированию. Заболевание позиционируют как “паразитарный рак” и диагностируют, как правило, на поздних стадиях. Результаты ОТП при нерезектабельных формах АП противоречивы, а серии представленных операций малочисленны по причине редкости заболевания $[6,7]$. Большинство авторов сходятся во мнении, что эти вмешательства технически чрезвычайно трудны в силу больших размеров 
пораженного органа и паразитарной инвазии в окружающие ткани. В свою очередь это требует существенного расширения объема операции резекции НПВ, смежных с печенью органов, сложных пластических и реконструктивных этапов $[6,8]$. По субъективным ощущениям у всех пациентов группы АП процесс мобилизации органа и гепатэктомии сопровождался наибольшими техническими трудностями, обусловленными указанными причинами. Нельзя не отметить, что зачастую эти пациенты физически истощены, инвалидизированы, имеют негативный опыт неоднократных паллиативных вмешательств [6, 8, 9]. В силу перечисленных обстоятельств пери- и послеоперационное лечение таких пациентов представляет серьезные трудности. По данным исследователей, ранняя послеоперационная летальность составляет 20\%, однолетняя выживаемость - 77\%, при этом бактериальная и грибковая инфекция является основной причиной смерти [8]. В обсуждаемом материале однолетняя ОВ в этой группе пациентов составила $62,5 \%$, и мы солидарны с авторами [8] в оценке причин неудовлетворительных результатов ОТП - все летальные исходы в этой группе были обусловлены септическими осложнениями. Вместе с тем публикации последних лет свидетельствуют о высокой эффективности ОТП с хорошими ближайшими и отдаленными результатами $[4,10]$.

Эпителиоидная гемангиоэндотелиома печени - редкая сосудистая опухоль, развивающаяся из клеток эндотелия и характеризующаяся неопределенным индексом малигнизации [3, 11]. Течение ЭГ может варьировать от пассивного до крайне агрессивного. В большинстве наблюдений ЭГ характеризуется билобарным, мультифокальным поражением печени, примерно у 25-36\% пациентов выявляют отдаленные метастазы [11]. По уже указанным причинам ЭГ диагностируют, как правило, на поздних стадиях, резектабельность опухоли отмечают не более чем в $10 \%$ наблюдений. ТП является операцией выбора при диффузной форме заболевания и характеризуется хорошими показателями БРВ и ОВ, в том числе у пациентов с внепеченочным распространением [3, 12]. Предикторами неблагоприятного прогноза считают макроваскулярную инвазию, метастазы в регионарные лимфатические узлы и трансплантацию, выполненную ранее 6 мес от момента установления диагноза ЭГ [3]. Важнейшим вопросом отбора пациентов на ОТП является корректная предоперационная дифференциальная диагностика между ЭГ и гемангиосаркомой, прогноз выживаемости при которой крайне неблагоприятный $[12,13]$. Гистологическое исследование остается основным, но, как правило, ретроспективным методом диагностики. Существующие методы иммуногисто- химического анализа (vWF, CD31, CD34) могут быть использованы в диагностике ЭГ, однако они остаются малодоступными. В обсуждаемом исследовании предоперационная биопсия выполнена 3 из 4 пациентов, что, однако, не повлияло на долгосрочные результаты. Однолетняя БРВ и ОВ составили 50 и 100\%, трехлетняя БРВ и ОВ - 50\%, что также свидетельствует о сложности предоперационной диагностики ЭГ, требующей более совершенных методов.

Существующий дефицит донорских органов и большой риск прогрессирования онкологического заболевания после ОТП являются главными сдерживающими факторами в расширении показаний к вмешательству у пациентов с нерезектабельными опухолями. ОТП по-прежнему не рекомендована при ХЦР в связи с плохим прогнозом [2, 14, 15]. Кроме того, трупная ТП не всегда осуществима в связи с требованиями, предъявляемыми к системе органного донорства, ориентированной на региональность распределения. Гипотермическая РП ex situ, ex vivo является успешной демонстрацией применения трансплантационных технологий в хирургии печени, с помощью которых в ряде наблюдений возможно решение указанных задач без использования донорского органа [16, 17]. Наиболее часто ЭРиА показана при опухолях и паразитарных заболеваниях печени, вовлекающих гепатикокавальный конфлюенс. Согласно собственному опыту, выбор ЭРиА в качестве метода лечения был обусловлен внепеченочным распространением АП $(n=1)$, ХЦР $(n=1)$ и отсутствием юридической возможности включения пациента в лист ожидания трупной печени $(n=2)$. Экстракорпоральным резекциям присуща большая частота серьезных послеоперационных осложнений (порядка 40\%), летальность варьирует от 6,5 до 28\% [16-18]. Важнейшими условиями успешной ЭРиА являются достаточный объем ( $\geqslant 35 \%$ от исходного) и сохранные функциональные резервы остающейся паренхимы печени. Несмотря на то что всем нашим пациентам выполняли до операции КТ-волюметрию, фатальная послеоперационная печеночная недостаточность, развившаяся в 1 наблюдении, была обусловлена сниженными функциональными резервами при достаточном объеме культи печени $(>45 \%)$.

\section{Заключение}

Некоторые нерезектабельные поражения печени могут быть успешно излечены трансплантацией или, реже, экстракорпоральной резекцией в условиях гипотермии. Наибольшему риску в раннем послеоперационном периоде подвержены пациенты с поздними формами альвеококкоза, поскольку их состояние исходно осложнено такими факторами, как истощение 
и хроническая инфекция. Лечение альвеококкоза печени следует начинать в специализированных гепатобилиарных центрах, имеющих опыт трансплантации. Это существенно увеличит радикальность лечения и избавит пациентов от паллиативных операций, не улучшающих ни качество жизни, ни результаты будущих хирургических вмешательств. Трансплантации печени при эпителиоидной гемангиоэндотелиоме должно предшествовать тщательное обследование, сводящее к минимуму вероятность раннего рецидива. ОТП при нерезектабельной ГЦК, развившейся вне фонового заболевания печени, характеризуется удовлетворительной безрецидивной и общей выживаемостью. Экстракорпоральные РП рекомендованы в отдельных клинических ситуациях, хирургическая и анестезиологическая бригады должны иметь опыт проведения трансплантаций печени.

\section{Участие авторов}

Новрузбеков М.С. - концепция и дизайн исследования, сбор и обработка материала, обработка данных литературы, написание текста, редактирование, утверждение окончательного варианта статьи, ответственность за целостность всех частей статьи.

Олисов О.Д. - концепция и дизайн исследования, сбор и обработка материала, обработка данных литературы, написание текста, редактирование, утверждение окончательного варианта статьи, ответственность за целостность всех частей статьи.

Гуляев В.А. - сбор и обработка материала.

Луцык К.Н. - сбор и обработка материала.

Магомедов К.М. - сбор и обработка материала.

\section{Authors participation}

Novruzbekov M.S. - concept and design of the study, collection and analysis of data, literature analysis, writing text, editing, approval of the final version of the article, responsibility for the integrity of all parts of the article.

Olisov O.D. - concept and design of the study, collection and analysis of data, literature analysis, writing text, editing, approval of the final version of the article, responsibility for the integrity of all parts of the article.

Guliaev V.A. - collection and analysis of data.

Lutsyk K.N. - collection and analysis of data.

Magomedov K.M. - collection and analysis of data.

\section{Список литературы}

1. Mehrabi A., Fonouni H., Golriz M., Hofer S., Hafezi M., Rahbari N.N., Weitz J., Büchler M.W., Schmidt J. Hypothermic ante situm resection in tumors of the hepatocaval confluence. Dig. Surg. 2011; 28 (2): 100-108. https://doi.org/10.1159/000323818.

2. European Association for the Study of the Liver. EASL clinical practice guidelines: liver transplantation. J. Hepatol. 2016; 64 (2): 433-485. https://doi.org/10.1016/j.jhep.2015.10.006.

3. Lai Q., Feys E., Karam V., Adam R., Klempnauer J., Oliverius M., Mazzaferro V., Pascher A., Remiszewski P., Isoniemi H., Pirenne J., Foss A., Ericzon B.G., Markovic S., Lerut J.P.
Hepatic epithelioid hemangioendothelioma and adult liver transplantation: proposal for a prognostic score based on the analysis of the ELTR-ELITA registry. Transplantation. 2017; 101 (3): 555-564. https://doi.org/10.1097/TP.0000000000001603.

4. Поршенников И.А., Быков А.Ю., Павлик В.Н., Карташов А.С., Щёкина Е.Е., Коробейникова М.А., Юшина Е.Г. Трансплантации и радикальные резекции печени с реконструкциями сосудов при распространенном альвеококкозе. Анналы хирургической гепатологии. 2016; 21 (2): 11-24. https://doi.org/10.16931/1995-5464.2016211-24.

5. Mergental H., Adam R., Ericzon B.G., Kalicinski P., Mühlbacher F., Höckerstedt K., Klempnauer J.L., Friman S., Broelsch C.E., Mantion G., Fernandez-Sellez C., van Hoek B., Fangmann J., Pirenne J., Muiesan P., Königsrainer A., Mirza D.F., Lerut J., Detry O., Le Treut Y.P., Mazzaferro V., Löhe F., Berenguer M., Clavien P.A., Rogiers X., Belghiti J., Kóbori L., Burra P., Wolf P., Schareck W., Pisarski P., Foss A., Filipponi F., Krawczyk M., Wolff M., Langrehr J.M., Rolles K., Jamieson N., Hop W.C., Porte R.J. Liver transplantation for unresectable hepatocellular carcinoma in normal livers. J. Hepatol. 2012; 57 (2): 297-305. https://doi.org/10.1016/j.jhep.2012.03.022.

6. Salm L.A., Lachenmayer A., Perrodin S.F., Candinas D., Beldi G. Surgical treatment strategies for hepatic alveolar echinococcosis. Food and Waterborne. Parasitology. 2019; 5 (15): e00050. https://doi.org/10.1016/j.fawpar.2019.e00050.

7. Li F., Yang M., Li B., Yan L., Zen Y., Wen T., Zao J. Initial clinical results of orthotopic liver transplantation for hepatic alveolar echinococcosis. Liver Transpl. 2007; 13 (6): 924-926. https://doi.org/10.1002/lt.21187.

8. Koch S., Bresson-Handi S., Miguet J.P., Crumbach J.P., Gillet M., Mantion G.-A., Heyd B., Vuitton D.A., Minello A., Kurtz S. Experience of liver transplantation for incurable alveolar echinococcosis: a 45-case European collaborative report. Transplantaton. 2003; 75 (6): 856-863. https://doi.org/10.1097/01.TP.0000054230.63568.79.

9. Pielok L., Karczewski M., Cierach W., Zmora P., Lenartowicz E., Stefaniak J. Portal hypertension as a result of the incomplete surgically treated advanced alveolar echinococcosis: a case description. BMC Gastroenterol. 2020; 20 (1): 176. https://doi.org/10.1186/s12876-020-01320-0.

10. Артемьев А.И., Найденов Е.В., Забежинский Д.А., Губарев К.К., Колышев И.Ю., Рудаков В.С., Шабалин М.В. Трансплантация печени при нерезектабельном альвеококкозе печени. Современные технологии в медицине. 2017; 9 (1): 123-128. https://doi.org/10.17691/stm2017.9.1.16.

11. Hu H.J., Jin Y.W., Jing Q.Y., Shrestha A., Cheng N.S., Li F.Y. Hepatic epithelioid hemangioendothelioma: dilemma and challenges in the preoperative diagnosis. World J. Gastroenterol. 2016; 22 (41): 9247-9250. https://doi.org/10.3748/wjg.v22.i41.9247.

12. Поршенников И.А., Быков А.Ю., Павлик В.Н., Щекина Е.Е., Белобородов К.Ю., Грязнов К.А., Лащенкова Е.В., Павлова Т.Н. Трансплантация печени при эпителиоидной гемангиоэндотелиоме с канцероматозом брюшной полости: клиническое наблюдение. Вестник трансплантологии и искусственных органов. 2013; 15 (4): 104-111.

13. van Rosmalen B.V., Verheij J., Phoa S.S.K.S., van Gulik T.M. Hepatic epithelioid haemangioendothelioma (HEHE): a diagnostic dilemma between haemangioma and angiosarcoma. BMJ Case Rep. 2017; 2017: bcr-2017-220687. https://doi.org/10.1136/bcr-2017-220687

14. Weber S.M., Ribero D., OReilly E.M., Kokudo N., Miyazaki M., Pawlik T.M. Intrahepatic cholangiocarcinoma: expert consensus 
statement. HPB (Oxford). 2015; 17 (8): 669-680.

https://doi.org/10.1111/hpb.12441.

15. Vogel A., Wege H., Caca K., Nashan B., Neuman U. The diagnosis and treatment of cholangiocarcinoma. Dtsch. Arztebl. Int. 2014; 111 (44): 748-754. https://doi.org/10.3238/arztebl.2014.0748.

16. Tuxun T., Aini A., Li Y.P., Apaer S., Zhang H., Li T., Aji T., Yimiti Y., Zhao J.M., Shao Y.M., Wen H. Systematic review and meta-analysis of feasibility, safety, and efficacy of ex situ liver resection and autotransplantation. Zhonghua Yi Xue Za Zhi. 2016; 96 (28): 2251-2257. https://doi.org/10.1016/j.hpb.2016.02.075.

17. Ye Q., Senninger N. The consensus on liver autotransplantation from an international panel of experts. Hepatobiliary Pancreat. Dis. Int. 2017; 16 (1): 10-16. https://doi.org/10.1016/s1499-3872(16)60175-3.

18. Yang X., Qiu Y., Huang B., Wang W., Shen S., Feng X., Wei Y., Lei J., Zhao J., Li B., Wen T., Yan L. Novel techniques and preliminary results of ex vivo liver resection and autotransplantation for end-stage hepatic alveolar echinococcosis: a study of 31 cases. Am. J. Transplant. 2018; 18 (7): 1668-1679. https://doi.org/10.1111/ajt.14621.

\section{References}

1. Mehrabi A., Fonouni H., Golriz M., Hofer S., Hafezi M., Rahbari N.N., Weitz J., Büchler M.W., Schmidt J. Hypothermic ante situm resection in tumors of the hepatocaval confluence. Dig. Surg. 2011; 28 (2): 100-108. https://doi.org/10.1159/000323818.

2. European Association for the Study of the Liver. EASL clinical practice guidelines: liver transplantation. J. Hepatol. 2016; 64 (2): 433-485. https://doi.org/10.1016/j.jhep.2015.10.006.

3. Lai Q., Feys E., Karam V., Adam R., Klempnauer J., Oliverius M., Mazzaferro V., Pascher A., Remiszewski P., Isoniemi H., Pirenne J., Foss A., Ericzon B.G., Markovic S., Lerut J.P. Hepatic epithelioid hemangioendothelioma and adult liver transplantation: proposal for a prognostic score based on the analysis of the ELTR-ELITA registry. Transplantation. 2017; 101 (3): 555-564. https://doi.org/10.1097/TP.0000000000001603.

4. Porshennikov I.A., Bykov A.Yu., Pavlik V.N., Kartashov A.S., Shchekina E.E., Korobeynikova M.A., Yushina E.G. Liver transplantation and liver resection with vascular reconstruction for advanced alveococcosis. Annaly khirurgicheskoy gepatologii $=$ Annals of HPB Surgery. 2016; 21 (2): 11-24. (In Russian) https://doi.org/10.16931/1995-5464.2016211-24.

5. Mergental H., Adam R., Ericzon B.G., Kalicinski P., Mühlbacher F., Höckerstedt K., Klempnauer J.L., Friman S., Broelsch C.E., Mantion G., Fernandez-Sellez C., van Hoek B., Fangmann J., Pirenne J., Muiesan P., Königsrainer A., Mirza D.F., Lerut J., Detry O., Le Treut Y.P., Mazzaferro V., Löhe F., Berenguer M., Clavien P.A., Rogiers X., Belghiti J., Kóbori L., Burra P., Wolf P., Schareck W., Pisarski P., Foss A., Filipponi F., Krawczyk M., Wolff M., Langrehr J.M., Rolles K., Jamieson N., Hop W.C., Porte R.J. Liver transplantation for unresectable hepatocellular carcinoma in normal livers. J. Hepatol. 2012; 57 (2): 297-305. https://doi.org/10.1016/j.jhep.2012.03.022.

6. Salm L.A., Lachenmayer A., Perrodin S.F., Candinas D., Beldi G. Surgical treatment strategies for hepatic alveolar echinococcosis. Food and Waterborne. Parasitology. 2019; 5 (15): e00050. https://doi.org/10.1016/j.fawpar.2019.e00050.
7. Li F., Yang M., Li B., Yan L., Zen Y., Wen T., Zao J. Initial clinical results of orthotopic liver transplantation for hepatic alveolar echinococcosis. Liver Transpl. 2007; 13 (6): 924-926. https://doi.org/10.1002/lt.21187.

8. Koch S., Bresson-Handi S., Miguet J.P., Crumbach J.P., Gillet M., Mantion G.-A., Heyd B., Vuitton D.A., Minello A., Kurtz S. Experience of liver transplantation for incurable alveolar echinococcosis: a 45-case European collaborative report. Transplantaton. 2003; 75 (6): 856-863.

https://doi.org/10.1097/01.TP.0000054230.63568.79.

9. Pielok L., Karczewski M., Cierach W., Zmora P., Lenartowicz E., Stefaniak J. Portal hypertension as a result of the incomplete surgically treated advanced alveolar echinococcosis: a case description. BMC Gastroenterol. 2020; 20 (1): 176. https://doi.org/10.1186/s12876-020-01320-0.

10. Artemyev A.I., Naydenov E.V., Zabezhinsky D.A., Gubarev K.K., Kolyshev I.Y., Rudakov V.S., Shabalin M.V. Liver transplantation for unresectable alveolar echinococcosis. Sovremennye tekhnologii $v$ meditsine $=$ Modern technologies in Medicine. 2017; 9 (1): 123128. https://doi.org/10.1186/s12876-020-01320-0 (In Russian)

11. Hu H.J., Jin Y.W., Jing Q.Y., Shrestha A., Cheng N.S., Li F.Y. Hepatic epithelioid hemangioendothelioma: dilemma and challenges in the preoperative diagnosis. World J. Gastroenterol. 2016; 22 (41): 9247-9250.

https://doi.org/10.3748/wjg.v22.i41.9247.

12. Porshennikov I.A., Bykov A.Yu., Pavlik V.N., Shchekina E.E., Beloborodov K.Yu., Gryaznov K.A., Lashchenkova E.V., Pavlova T.N. Liver transplantation for epithelioid hemahgioendotelioma with diffuse intraperitoneal metastases: a case report. Russian Journal of Transplantology and Artificial Organs. 2013; 15 (4): 104-111. (In Russian)

13. van Rosmalen B.V., Verheij J., Phoa S.S.K.S., van Gulik T.M. Hepatic epithelioid haemangioendothelioma (HEHE): a diagnostic dilemma between haemangioma and angiosarcoma. BMJ Case Rep. 2017; 2017: bcr-2017-220687. https://doi.org/10.1136/bcr-2017-220687

14. Weber S.M., Ribero D., OReilly E.M., Kokudo N., Miyazaki M., Pawlik T.M. Intrahepatic cholangiocarcinoma: expert consensus statement. HPB (Oxford). 2015; 17 (8): 669-680. https://doi.org/10.1111/hpb.12441.

15. Vogel A., Wege H., Caca K., Nashan B., Neuman U. The diagnosis and treatment of cholangiocarcinoma. Dtsch. Arztebl. Int. 2014; 111 (44): 748-754. https://doi.org/10.3238/arztebl.2014.0748.

16. Tuxun T., Aini A., Li Y.P., Apaer S., Zhang H., Li T., Aji T., Yimiti Y., Zhao J.M., Shao Y.M., Wen H. Systematic review and meta-analysis of feasibility, safety, and efficacy of ex situ liver resection and autotransplantation. Zhonghua Yi Xue Za Zhi. 2016; 96 (28): 2251-2257. https://doi.org/10.1016/j.hpb.2016.02.075.

17. Ye Q., Senninger N. The consensus on liver autotransplantation from an international panel of experts. Hepatobiliary Pancreat. Dis. Int. 2017; 16 (1): 10-16. https://doi.org/10.1016/s1499-3872(16)60175-3.

18. Yang X., Qiu Y., Huang B., Wang W., Shen S., Feng X., Wei Y., Lei J., Zhao J., Li B., Wen T., Yan L. Novel techniques and preliminary results of ex vivo liver resection and autotransplantation for end-stage hepatic alveolar echinococcosis: a study of 31 cases. Am. J. Transplant. 2018; 18 (7): 1668-1679. https://doi.org/10.1111/ajt.14621. 


\section{Сведения об авторах [Authors info]}

Новрузбеков Мурад Сафтарович - доктор мед. наук, заведующий научным отделением трансплантации печени НИИ СП им. Н.В. Склифосовского. https://orcid.org/0000-0002-6362-7914. E-mail: N.m.s@bk.ru

Олисов Олег Даниелович - канд. мед. наук, старший научный сотрудник отделения трансплантации печени НИИ СП им. Н.В. Склифосовского. https://orcid.org/0000-0002-0691-5581. E-mail: Dr.Olisov@gmail.com

Гуляев Владимир Алексеевич - доктор мед. наук, ведущий научный сотрудник отделения трансплантации почки и поджелудочной железы НИИ СП им. Н.В. Склифосовского. https://orcid.org/0000-0001-8650-0855. E-mail: GuluaevVA@ sklif.mos.ru

Луцык Константин Николаевич - канд. мед. наук, заведующий операционным блоком центра трансплантации печени НИИ СП им. Н.В. Склифосовского. https://orcid.org/0000-0003-2305-4055. E-mal: S.urg@mail.ru

Магомедов Кубай Магомедович - врач-хирург отделения трансплантации печени НИИ СП им. Н.В. Склифосовского. https://orcid.org/0000-0002-5057-6628. E-mail: Kubay.agul@gmail.com

Для корреспонденции *: Новрузбеков Мурад Сафтарович - 129090, Москва, Большая Сухаревская пл., д. 3/5, Российская Федерация. Тел.: 8-495-628-35-02. E-mail: N.m.s@bk.ru

Олисов Олег Даниелович - 129090, Москва, Большая Сухаревская пл., д. 3/5, Российская Федерация.

Тел.: 8-495-628-35-02. E-mail: Dr.Olisov@gmail.com

Murad S. Novruzbekov - Doct. of Sci. (Med.), Head of the Scientific Department of Liver Transplantation of the Sklifosovsky Research Institute for Emergency Medicine, Moscow. https://orcid.org/0000-0002-6362-7914. E-mail: N.m.s@bk.ru

Oleg D. Olisov - Cand. of Sci. (Med.), Senior Researcher of the Liver Transplant Department of the Sklifosovsky Research Institute for Emergency Medicine, Moscow. https://orcid.org/0000-0002-0691-5581. E-mail: Dr.Olisov@gmail.com

Vladimir A. Guliaev - Doct. of Sci. (Med.), Leading Researcher of the Kidney and Pancreas Transplantation Department of the Sklifosovsky Research Institute for Emergency Medicine, Moscow. https://orcid.org/0000-0001-8650-0855. E-mail: GuluaevVA@sklif.mos.ru

Konstantin N. Lutsyk - Cand. of Sci. (Med.), Head of the Surgery Suite of the Liver Transplantation Department, Sklifosovsky Research Institute for Emergency Medicine, Moscow. https://orcid.org/0000-0003-2305-4055. E-mal: S.urg@mail.ru

Kubay M. Magomedov - Surgeon of the Liver Transplantation Department, Sklifosovsky Research Institute for Emergency Medicine, Moscow. https://orcid.org/0000-0002-5057-6628. E-mail: kubay.agul@gmail.com

For correspondence $^{*}$ : Murad S. Novruzbekov - 3/5, Bolshaya Suharevskaya sq., Moscow, 129090, Russian Federation. Phone: 8-495-628-35-02. E-mail: N.m.s@bk.ru

Oleg D. Olisov - 3/5, Bolshaya Suharevskaya sq., Moscow, 129090, Russian Federation. Phone: 8-495-628-35-02.

E-mail: Dr.Olisov@gmail.com

Статья поступила в редакцию журнала 09.09.2020. Принята к публикации 29.09.2020.

Received 9 September 2020. Accepted for publication 29 September 2020. 